\title{
Assembling Native Elementary Cellulose Nanofibrils via a Dynamic and Spatially Confined Functionalization
}

Marco Beaumont, ${ }^{1 \# *}$ Blaise L. Tardy, ${ }^{2 \#}$ Guillermo Reyes, ${ }^{2}$ Tetyana V. Koso, ${ }^{3}$ Elisabeth Schaubmayr, ${ }^{1}$ Paul Jusner, ${ }^{1}$ Alistair W. T. King, ${ }^{3}$ Raymond R. Dagastine, ${ }^{4}$ Antje Potthast,${ }^{1}$ Orlando J. Rojas, ${ }^{2,5 *}$ and Thomas Rosenau ${ }^{1,6 *}$

${ }^{1}$ Department of Chemistry, Institute of Chemistry for Renewable Resources, University of Natural Resources and Life Sciences Vienna (BOKU), Konrad-Lorenz-Straße 24, A-3430 Tulln, Austria.

${ }^{2}$ Department of Bioproducts and Biosystems, School of Chemical Engineering, Aalto University, P.O. Box 16300, Espoo FI-00076, Finland.

${ }^{3}$ Materials Chemistry Division, Department of Chemistry, University of Helsinki, AI Virtasen aukio 1, FI-00560 Helsinki, Finland.

${ }^{4}$ Department of Chemical \& Biomolecular Engineering; The University of Melbourne, Victoria, Australia.

${ }^{5}$ Bioproducts Institute, Department of Chemical \& Biological Engineering, Chemistry and Wood Science, The University of British Columbia, Vancouver, BC V6T 1Z3, Canada.

${ }^{6}$ Johan Gadolin Process Chemistry Centre, Åbo Akademi University, Porthansgatan 3, Åbo/Turku FI-20500, Finland.

\#These authors contributed equally to this work. *Corresponding authors: $\underline{\text { thomas.rosenau@boku.ac.at, orlando.rojas@ubc.ca, marcobeaumont1@gmail.com }}$ KEYWORDS: Transesterification, functional nanoparticles, biomass colloids, Individualized nanofibers, reversible succinylation, imidazole chemistry 


\begin{abstract}
Selective surface modification of bio-based fibers affords effective individualization and functionalization into nanomaterials, as shown by the TEMPO-mediated oxidation. However, such route leads to changes of the native surface chemistry, affecting interparticle interactions and limiting the full exploitation of the intrinsic supermaterial properties. Here we introduce a methodology to extract elementary cellulose fibrils by treatment of biomass with $\mathrm{N}$ succinylimidazole, achieving spatially confined (regioselective modification of $\mathrm{C} 6-\mathrm{OH}$ ) and dynamic surface functionalization. No polymer degradation or crosslinking nor changes in crystallinity occur under the mild conditions of the process and the modification is fully reversible, which offers a significant opportunity for the reconstitution of the interfaces back to the native states, chemically and structurally. Consequently, access to 3D structuring of native elementary cellulose fibrils is made possible with the same supramolecular features as the biosynthesized fibers, which is required to unlock the full potential of cellulose as a sustainable building block.
\end{abstract}




\section{Introduction}

New bio-based feedstock streams are needed to develop sustainable materials that surpass in performance the prevalent synthetic counterparts. In this regard, the isolation from biomass of native structural components of high intrinsic cohesion and defined morphology presents a unique opportunity. ${ }^{1}$ The biogenesis of cellulose chains from synthase systems results in polymeric constructs with the highest strength reported to date. Driven by supramolecular interactions, tightly packed elementary fibrils are formed (diameter of approx. 3-4 nm) exhibiting a remarkable tensile strength and modulus, reaching values as high as $7 \mathrm{GPa}^{2,3}$ and $140 \mathrm{GPa},{ }^{4-6}$ respectively. They can be readily obtained from forestry, ocean and agricultural side-streams and their promise in high-performance sustainable materials has triggered great interest over the last decade. ${ }^{1,3}$ However, there is a standing need for new, green routes to reengineer the native cellulosic supramolecular interactions into macroscale materials, ideally in line with green chemistry and technology principles.

Mechanical fibrillation of the plant cell wall, following optional pretreatments (e.g., enzymatic), results in bundled of cellulose nanofibrils (CNFs) carrying residual hemicelluloses, which dominate most supramolecular interactions, given their higher surface activity and reactivity. 7,8 The most prominent chemical pretreatment that enables individualization into elementary fibrils is a regioselective modification by oxidation of the cellulose's primary $\mathrm{OH}$ groups, namely, TEMPO-mediated oxidation (2,2,6,6-tetramethylpiperidin-1-yl-oxyl being the oxidant). ${ }^{9}$ An alternative approach is the periodate oxidation and subsequent Pinnick oxidation, ${ }^{10}$ which converts the secondary alcohol groups of cellulose into carboxyl moieties. While these modifications are commonly used, they also present major drawbacks, including those related to chemical degradation and their irreversibility. For instance, complete surface modification of the nanofibrils by TEMPO-oxidation has been demonstrated to occur in alkaline media, ${ }^{11,12}$ causing a drastic decrease in molar mass, ${ }^{13}$ even observed after moderate oxidation conditions (in the range of $0.5 \mathrm{mmol} \mathrm{COOH} \mathrm{g}{ }^{-1}$ of CNFs). ${ }^{14,15}$ These effects limit the mechanical performance and the corresponding prospects of the assembled materials. ${ }^{16}$ In addition, TEMPO-oxidation is irreversible, yielding nanofibrils with carboxylated surfaces, which prevent the strong interactions that is otherwise present in the native supramolecular structures. ${ }^{11}$ By contrast, cellulose esters can be cleaved by saponification, enabling the recovery of the intrinsic surface functionality and cohesive interactions. ${ }^{17}$ This latter observation inspired our inquiries, as presented in this discussion. 
Herein, cellulose fibers were deconstructed into elementary fibrils by regioselective modification with $N$-succinylimidazole (Figure 1). This surface modification is introduced on the entire fibril surface, with very high selectivity towards the primary hydroxyl group of cellulose. ${ }^{18-20}$ The method is mild and does not lead to dissolution nor influence the inherent physico-chemical properties such as crystallinity, and molar mass while preserving the morphology of the elementary, even at complete surface functionalization. Preserving such native properties are essential to maintain the excellent mechanical properties of cellulose., ${ }^{4,5}$ The negative carboxylate charge facilitates individualization into elementary nanofibrils, which can be processed, in a similar fashion as TEMPO-CNF, into an arbitrary shape, e.g. via extrusion, wet-spinning or film formation. ${ }^{1,21,22}$ Although for TEMPO-CNF the interfacial interactions were impacted by carboxyls groups, the succinyl ester moieties can be hydrolyzed ( saponified) under basic conditions to recover the pristine chemical structure of cellulose I, reaccessing its native supramolecular interactions. This offers a unique route to enhance processability of plant biomass into materials while recovering their performance potential. We describe the efficiency of the reaction, its regioselectivity, and reversibility by nuclear magnetic resonance, infrared spectroscopy, and gel permeation chromatography. The morphology of the elementary fibrils is then evaluated using atomic force microscopy and scanning electron microscopy, revealing the fibril morphology and size, which match that of the native fibrils. Finally, we demonstrate processing in aqueous media that leads to hydrogels, aerogels and films, the latter being compared for their mechanical properties before and after saponification. The introduced, new avenue to engineer cellulosic building blocks will unlock new opportunities in the fabrication of sustainable, high strength and light-weight materials. 


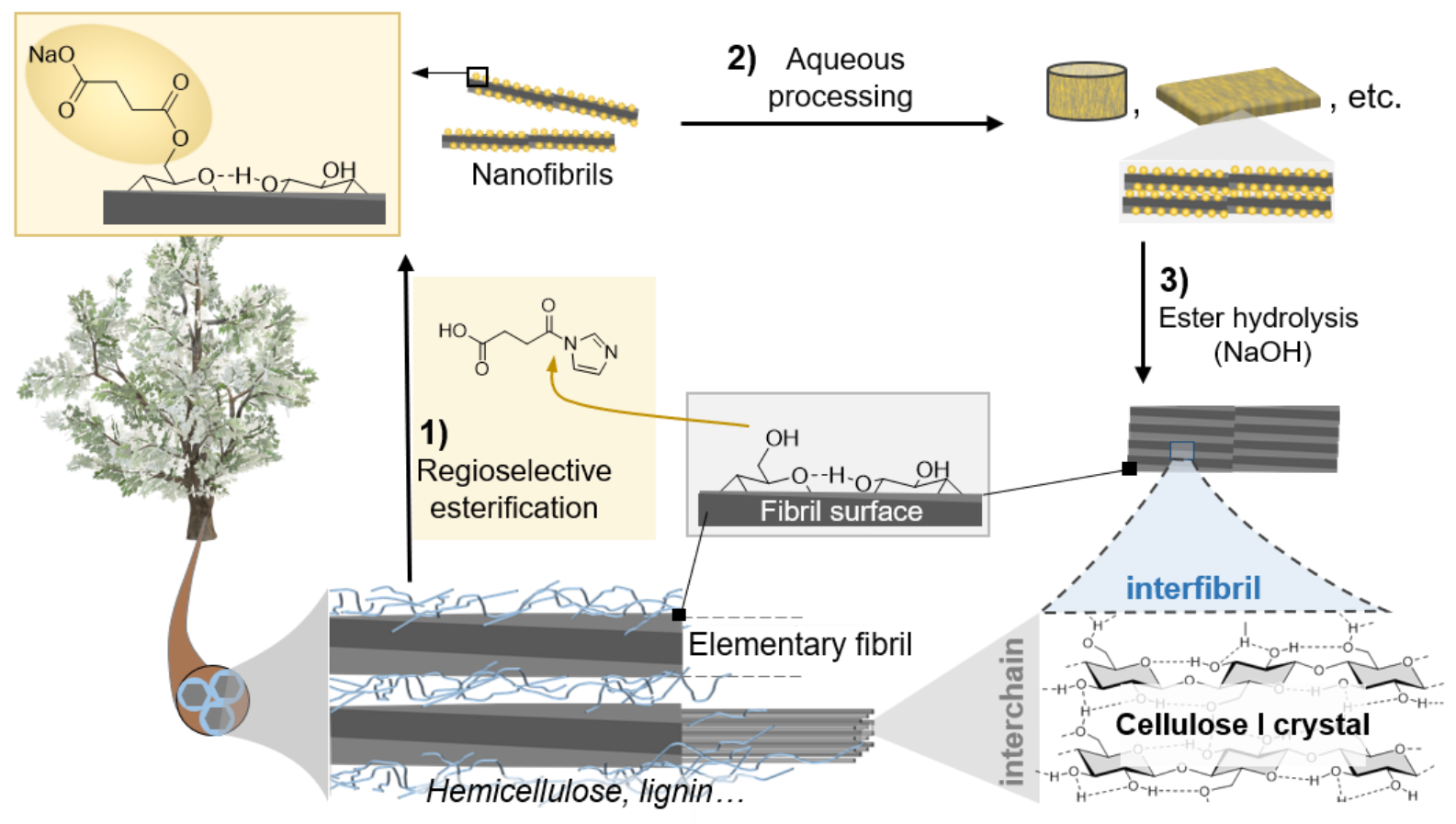

Figure 1. Elementary succinylated cellulose nanofibrils (C6SA-CNF) were produced from renewable cellulose fibers by regioselective esterification of the primary $\mathrm{C6}-\mathrm{OH}$ by reaction with $N$-succinyl imidazole followed by fibrillation in a high-pressure homogenizer (1). As is the case of conventional CNF, C6SA-CNF forms hydrogels of given shapes (2) but is amenable to removal of the installed groups by facile hydrolysis. Hence, 3D structures can be formed by the elementary fibrils of cellulose in its type I, the native cellulose allomorph (3). The hydrolysis treatment induces strong interfibrillar interactions, rivaling those present in the native cellulose I crystals.

\section{Results and Discussion}

The preparation of structural assemblies based on pristine individualized cellulose nanofibrils is shown schematically in Figure 1. The native cellulose fibers were regioselectively modified by succinylation of the surface C6-OH glucose repeating units. Thereby, CNF with the same selectivity (C6) and largely similar chemical functionality (succinate vs.carboxylate) as the well-known TEMPO-CNF were obtained. The esterification was mediated via a reactive acylimidazole intermediate, ${ }^{18-20}$ and can be applied directly to never-dried biomass, i.e., wet pulp fibers, commonly used for the preparation of typical CNF. ${ }^{10,12,18}$ as it is an acyl transfer rather than a classical esterification mechanism. 
The modification proceeded in an acetone/water solvent system in the presence of succinic anhydride (1.0 molar equivalents based on cellulose monomer unit) and imidazole (1.5 equivalents) that react in situ to form $N$-succinylimidazole (Figure 2A). The esterification was completed after approx. $6 \mathrm{~h}$, but we note that the final carboxylate content can be tailored by varying the reaction conditions (Figure S1). Successful introduction of the succinyl group was demonstrated by infrared spectroscopy, which confirmed the presence of the carbonyl bands at $1568 \mathrm{~cm}^{-1}$ and $1723 \mathrm{~cm}^{-1}$ (Figure 2B). The modified cellulose was directly compared with the starting material, never-dried cellulose fibers, which is referred to as 'reference'. The degree of substitution, determined by conductometric titration (Figure S2) was $0.25 \pm 0.02$, corresponding to a carboxylate content of $1.3 \pm 0.1 \mathrm{mmol} / \mathrm{g}$ (Table S1). These values were also confirmed by diffusion-edited liquid state NMR in the [ $\left.\mathrm{P}_{4444}\right][\mathrm{OAc}]: D M S O-\mathrm{d}_{6}$ solvent. $^{23,24}$ This analysis also afforded information on the regioselectivity of the modification (Figure 2C), ${ }^{19,20}$ and NMR peaks were assigned through the respective multiplicity-edited heteronuclear singlequantum correlation (HSQC) spectrum (Figure S3). The calculated total DS and that specific to the primary $\mathrm{C} 6-\mathrm{OH}$ were 0.24 and 0.22 , respectively, yielding a reaction regioselectivity of $92 \%$. Based on the crystallite size, we calculated the theoretically available number of C6-OH (Table S1), and concluded that the entire number of available C6-OH groups at the surface of the elementary fibrils was modified, which has been so far only possible by severe TEMPOoxidation. ${ }^{11}$ Solid-state NMR (Figure S4 and Figure 2E), was used to gather information on the fibril superstructure based on deconvolution of the $\mathrm{C} 4$ peak, composed of a chemically nonaccessible, crystalline core, and an accessible surface..$^{25,26}$ One of the peaks corresponding to the chemically accessible surface - shaded in dark grey and bronze for reference and C6SA$\mathrm{CNF}$, respectively - is clearly shifted upfield (from $83.2 \mathrm{ppm}$ to $82.5 \mathrm{ppm}$ ) upon surface modification. We assign this peak to $\mathrm{C} 4$ of the C6-succinylated glucopyranose surface units and further derive a homogeneous surface modification. In addition, it is clearly shown that the fibril superstructure and crystallinity (Table S1) is well preserved, and contrasts with NMR results of TEMPO-oxidized CNF (Figure S5). Compared to oxidative treatments, such as periodate or TEMPO-oxidation, the molar mass is not reduced (although changes occurred due to the introduction of succinyl groups, Figure 2D). The increase of the weight-averaged molar mass upon modification (Table S2) is the result of removal of hemicelluloses and/or low molar mass cellulose fractions; the former possibility, in fact, was confirmed by solid-state NMR measurements (Table S1).

The effect of the fibrillation degree on the rheological properties was assessed for C6SA-CNF and compared to that of TO-CNF (prepared under neutral conditions) ${ }^{14}$ (Figure S6), showing 
that C6SA-CNF underwent a slightly more extensive fibrillation. Moreover, we show that as other CNF types, C6SA-CNF exhibits strong shear-thinning and the rheological properties typical of a gel, due its dominating elastic behavior ( $G^{\prime}>G^{\prime}$ ') (Figure S7).
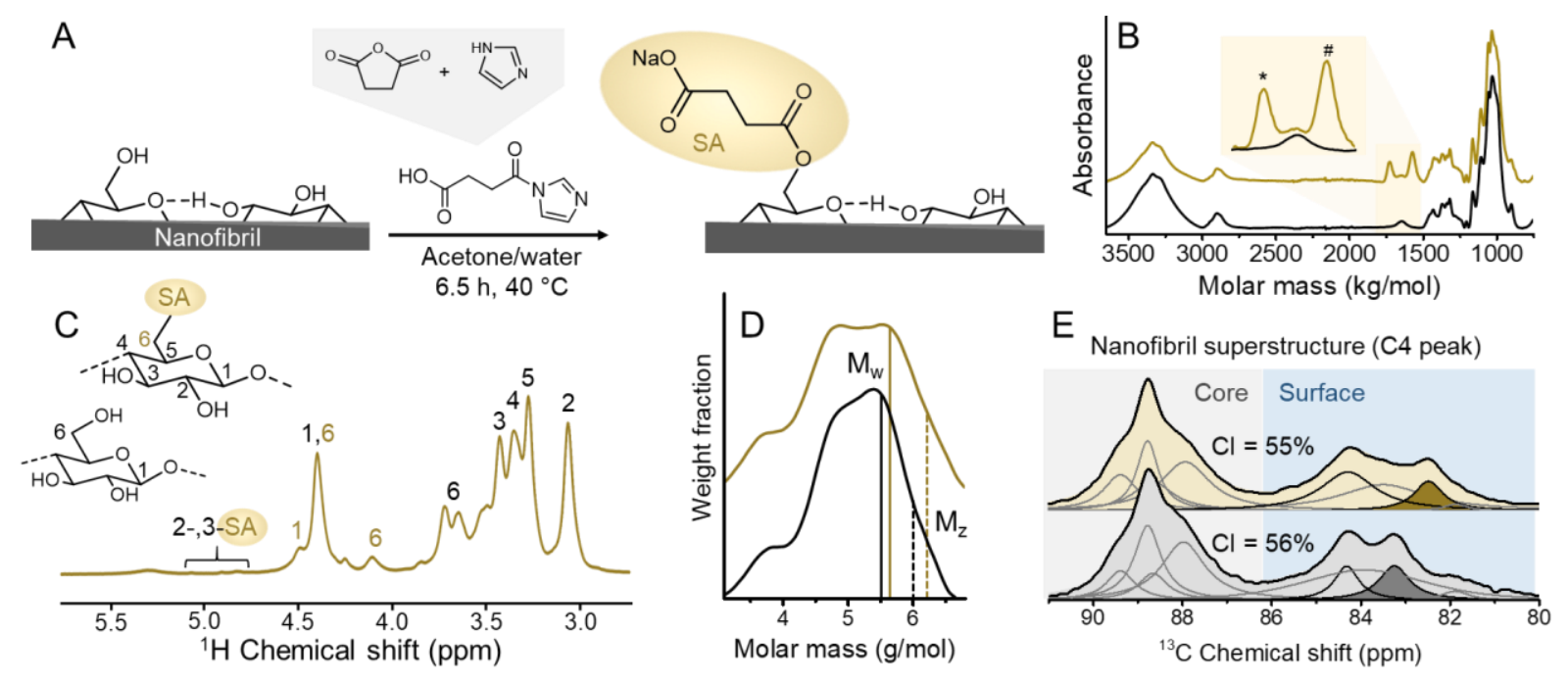

Figure 2. $N$-Succinyl imidazole, the acyl transfer agent, was produced in situ through reaction of imidazole and succinic anhydride (A). The wet cellulose fibers were esterified at $40{ }^{\circ} \mathrm{C}$ for $6.5 \mathrm{~h}$ in an acetone/water mixture to introduce the succinyl group onto the primary C6-OH of cellulose (92\% selectivity). The IR spectrum clearly demonstrates the successful introduction of the succinyl group $\left(* \lambda=1723 \mathrm{~cm}^{-1},{ }^{*} \lambda=1568 \mathrm{~cm}^{-1}\right.$ ) (B) and the regioselectivity was studied by solution-state nuclear magnetic resonance, showing only minor modification of the $\mathrm{C} 2$ - and $\mathrm{C} 3-\mathrm{OH}(\mathrm{C})$. The molar mass (both weight- and z-averaged) increased through introduction of the succinyl group (D). The superstructure and crystallinity index (CI), studied by solid-state NMR, was preserved, confirming that the reaction was confined to the accessible (amorphous) nanofibril surface $(\mathrm{E})$.

The morphology and dimensions of the nanofibrils were evaluated using atomic force microscopy (Figure 3). When observed, in the given scanning areas, that the fibrils appeared rather homogeneous (Figure 3A); meanwhile, the absence of bundling suggested complete individualization into elementary nanofibrils through our process. Figure 3A includes over 50 fibrils that were clearly individualized and the corresponding height profiles revealed rather uniform height values across the sample (Figure 3A2), e.g., a narrow height distribution (3.4 $\mathrm{nm} \pm 0.6 \mathrm{~nm}$, Figure S9). These values agree with those measured for elementary fibrils (also referred to as crystallite size) of approx. $4 \mathrm{~nm}$ (Table S1). This clearly indicates a complete fibrillation of the cellulose fiber into its elementary nanofibrils. Interestingly, a small fraction of the deposits on the surface were smaller fibrils with heights below $1 \mathrm{~nm}$ and lengths of ca. 
$20 \mathrm{~nm}$. These may be cellulosic fragments resulting from the pulping process, fibrillation treatment and/or residual hemicelluloses.
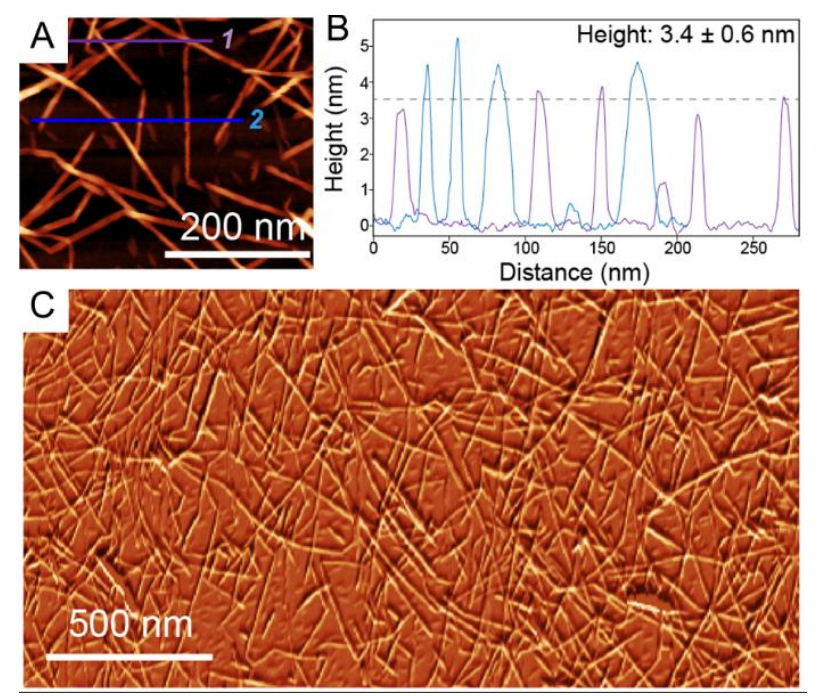

Figure 3 (A) Atomic force microscopy imaging of elementary fibrils of C6SA-CNF and (B) height profiles obtained for 9 fibrils. (C) The overview microscopy image indicates well dispersed and homogeneous fibrils $(\mathrm{C})$.

The chemistry used herein is nondestructive, which contrasts significantly with the oxidative routes conventionally used for modification or enhanced dispersion of nanocelluloses. Moreover, the introduced succinate ester is stable under conventional conditions (pH range 39). This enables a wide and versatile application range for C6SA-CNF. It is well known that ester groups are susceptible to hydrolysis, i.e., saponification. This is applicable to our modified $\mathrm{CNF}$, i.e., by treatment with $0.1 \mathrm{M} \mathrm{NaOH}$, yielding native elementary $\mathrm{CNF}$, which we submit to be similar to the natural form (herein referred to as nat-CNF) (Figure 4A). It can be reasonably proposed that pristine cellulose is restored after hydrolysis, upon removal of the succinate groups, as demonstrated by IR spectroscopy (Figure 4B) through the disappearance of the carbonyl bands and as also shown by gel permeation chromatography with multi-angle light scattering detection. Both weight- and z-average molar mass of the succinylated cellulose were clearly reduced upon saponification, returning to values similar to those of the reference sample, which is also well reflected in the respective molar mass distributions (Table S2 and Figure S8). Moreover, the conformation plot of nat-CNF (Figure 4C) showed that after hydrolytic treatment the shape and dependency of the radius of gyration and molar mass of the dissolved cellulose sample, returned to the initial, reference state. Generally, the alkaline treatment induced a crosslinking/gelation of the C6SA-CNF, similar to the behavior of 
carboxylated CNF under acidic conditions (protonation) or in presence of multivalent ions (ionic crosslinking through replacing $\mathrm{Na}^{+}$counterion).

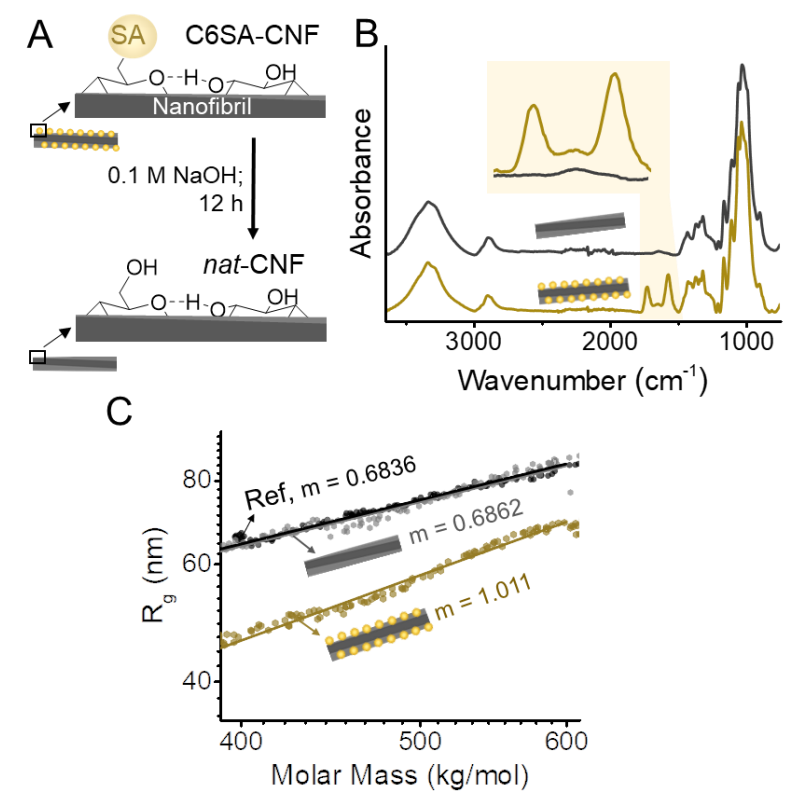

Figure 4. (A) Regioselective introduction of succinyl groups in C6SA-CNF (top-left) can be reversed by alkaline treatment $(0.1 \mathrm{M} \mathrm{NaOH}$, bottom-left), as shown by IR spectroscopy, through the disappearance of the carbonyl bands (B), as well as by the conformation plot from light scattering analysis $(C)$. The conformation plot (molar mass vs. radius of gyration, $\mathrm{R}_{\mathrm{g}}$ ) of the $\mathrm{NaOH}$-treated $\mathrm{C} 6 \mathrm{SA}-\mathrm{CNF}$ and its slope $(\mathrm{m})$ is almost identical compared to the native sample (Ref), confirming the reversible nature of the esterification.

The alkaline treatment can be conducted directly from C6SA-CNF in sodium form, or C6SACNF hydrogels prepared by ionic crosslinking. The properties of swollen networks of C6SACNF and TO-CNF, both processed into films, were then evaluated as a function of the conditions of the aqueous suspension (Figure S10). The films were swollen to equilibrium under various conditions and evaluated through the increase in thickness in relation to the dry film. In pure water, both C6SA-CNF and TO-CNF films swelled significantly, as shown by the 8.5- and 15-fold increase in thickness, respectively. The lower increase of C6SA-CNF is most probably related to the fact that the succinyl groups of C6SA-CNF are less hydrophilic compared to the carboxylate groups in TO-CNF. In contrast, the thickness increased significantly less in mild acidic conditions $(0.01 \mathrm{M} \mathrm{HCl})$, due to the protonation of the carboxylate groups and thereby induced gelation (due to lower electrostatic repulsion). In comparison, upon exposure to a base treatment $(0.1 \mathrm{M} \mathrm{NaOH})$, the thickness of TO-CNF films increased to a similar level as that in pure water (13-fold), while C6SA-CNF swelling was 
severely limited (3-fold), which is a result of the ester hydrolysis into nat-CNFs and the induced gelation.

The mechanical properties of the hydrogels (measured while immersed in the respective aqueous solution) were evaluated by tensile testing, Figure 5A-B, with representative tensile strain curves shown in Figure 5C. As expected, both TO-CNF and C6SA-CNF hydrogels showed a rather low mechanical strength when immersed in water, 0.3 and $2.2 \mathrm{MPa}$, respectively. The results are in line with the lower swelling of C6SA-CNF, which helps to resist the effect of water in the wet conditions. C6SA-CNF and TO-CNF films presented similar tensile strengths under acidic conditions, 12.5 and $20 \mathrm{MPa}$, respectively (Figure S11); the elastic modulus and toughness followed similar ranking. Upon $0.1 \mathrm{M} \mathrm{NaOH}$ treatment, the mechanical properties of the TO-CNF hydrogel were similar to those measured in water. In contrast, the tensile strength of $\mathrm{NaOH}$-treated C6SA-CNF (nat-CNF, Figure 5B) were of the same order as that of C6SA-CNF, $12 \mathrm{MPa}$, when the aqueous medium was changed to an acid medium (Figure S11). This demonstrates that the ester hydrolysis (saponification) induced physical crosslinking, due to the removal of the charged ester group, restoring the hydrogenbond network of native celluloses. The hydrogels were solvent-exchanged with acetone and supercritically dried and the resultant nanofibrillar network was observed by scanning electron microscopy. The network formed showed similar structures prior to and after removal of the succinyl groups. We speculate that the network formed after saponification resembles that of native cellulose nanofibrils (Figure 5D-E).

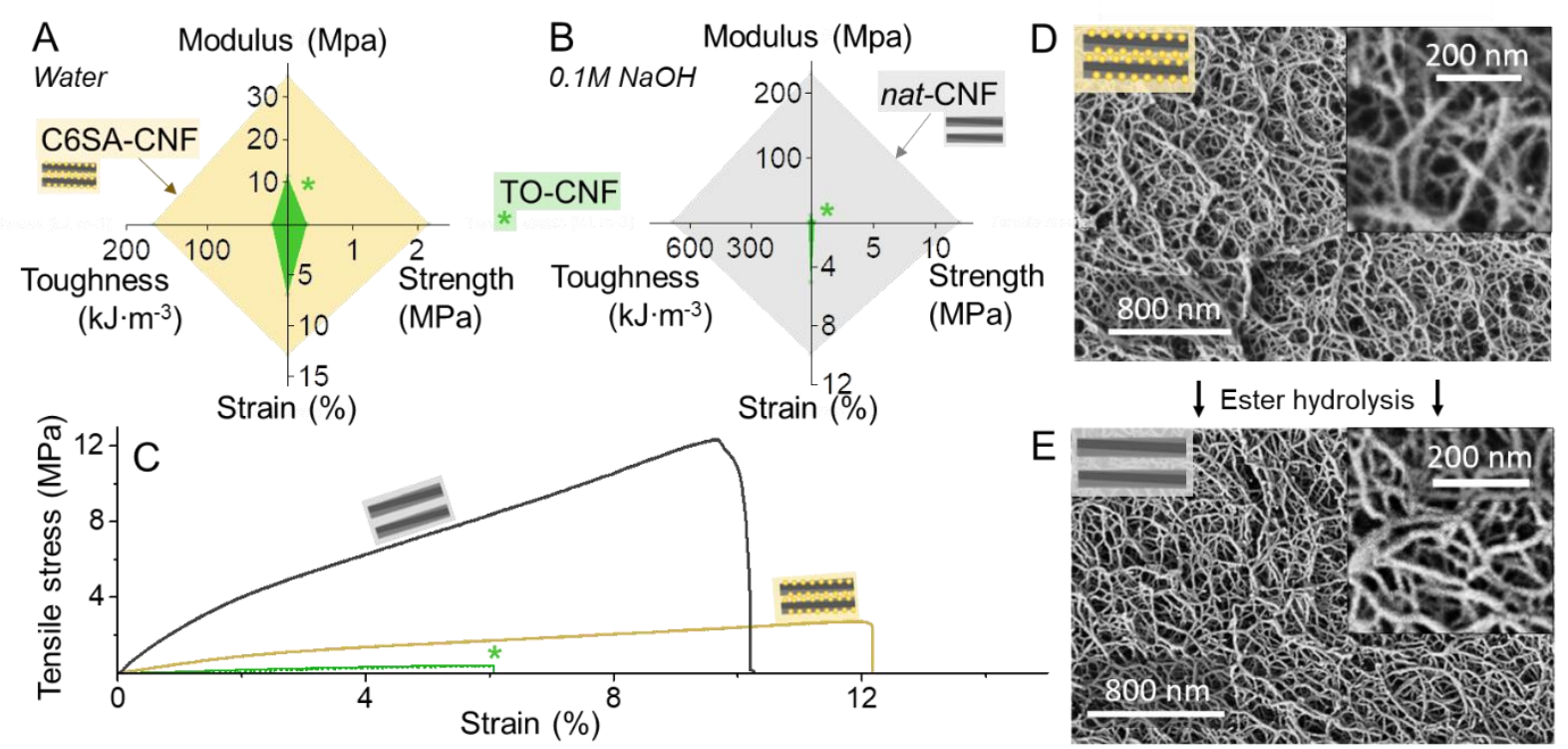

Figure 5 Mechanical performance of hydrogels produced from C6SA-CNF and TO-CNF, which were equilibrated in water prior (B) and after (A) treatment in $0.1 \mathrm{M} \mathrm{NaOH}$. C6SA-CNF 
exhibits stronger mechanical stability in water, which is especially pronounced in case of prior $\mathrm{NaOH}$ treatment, causing ester hydrolysis to yield nat-CNF. (C) Representative tensile profiles of the systems compared in A and B. Networks of C6SA-CNF (D) and nat-CNF (E) in an aerogel. The schematic figures highlight the possible nanofiber surface structure with and without prior $\mathrm{NaOH}$ treatment.

As shown in Figure 5a3, the supramolecular interactions in water, upon recovery of the native cellulose interfaces, resulted in an improved tensile strength, by ca. 6-fold, with a relatively small reduction of the strain at break, from $13 \%$ to $10 \%$. By favoring supramolecular interactions, upon drying, the strain at break was on the other hand reduced, from $3.2 \%$ to $1.8 \%$ (Figure 6). The mechanical performance of the respective dry films are compared in Figure 6. A significant increase in strength (a 2.5-fold increase, from $79 \mathrm{MPa}$ to $194 \mathrm{MPa}$ ) was realized. Likewise, the moduli increased from $7.9 \mathrm{GPa}$ to $19.4 \mathrm{GPa}$. This suggests that a significant improvement in the interfacial cohesion results from the recovery of the native supramolecular interactions, after saponification. With increased anisotropy, i.e., with increased coherence between the fibrils, one can expect further increase in strength of the formed films. 


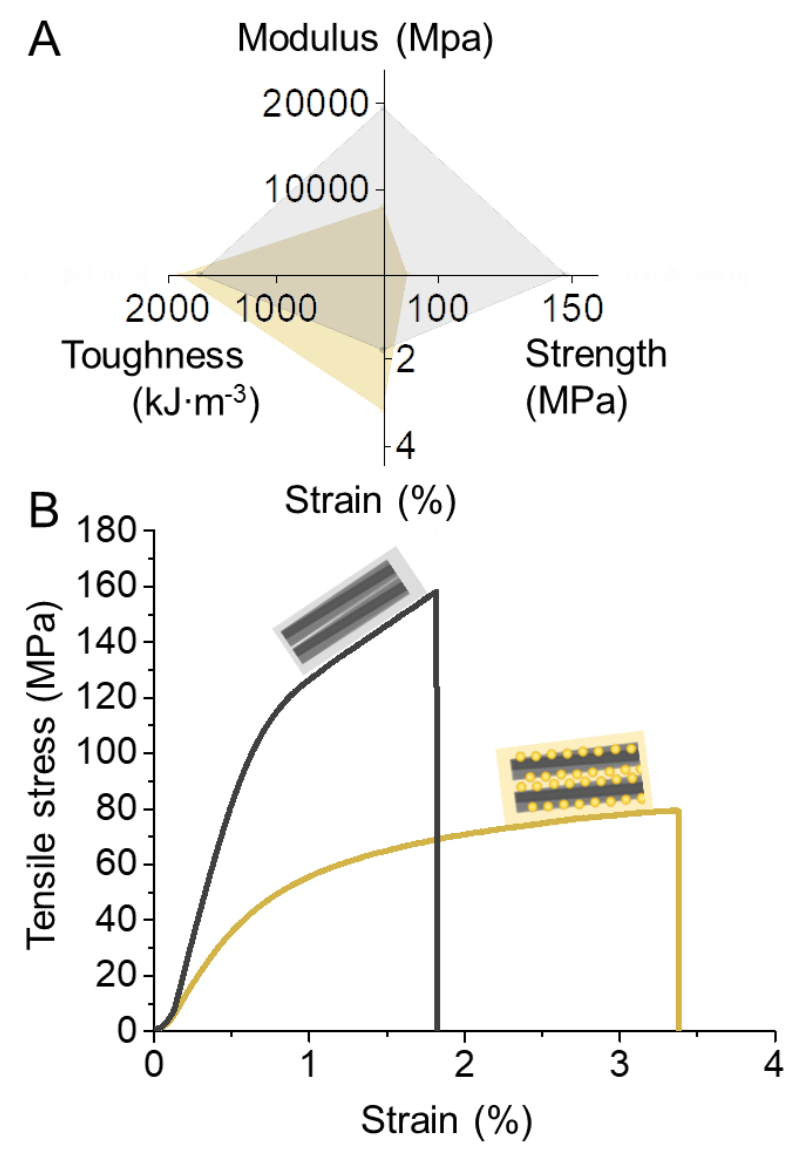

Figure 6 (A) Mechanical properties of dried, isotropic films of C6SA-CNF (yellow profile and shade) and nat-CNF (grey profile and shade). (B) Representative stress-strain curves for the samples indicate the suggested recovery of the native, interfibrillar cohesion of nat-CNF.

\section{Conclusions}

In conclusion, we have shown that our aqueous-based succinylation enables complete isolation of the elementary fibrils from wood fibers, without compromising the crystalline structure and degree of polymerization, within and on the surface of the nanofibrils. Moreover, we show that the proposed modification enables complete esterification of the available primary hydroxyl groups of the elementary fibril, i.e. full surface coverage, and thereby fibrillation into elementary nanofibrils. The resulting nanofibril dispersions could be processed using conventional approaches while making possible restoration of the native supramolecular interactions, by following a mild hydrolysis (saponification). The reversibility of the proposed functionalization enables structures comprising elementary nanofibrils exhibiting their more characteristics, which can overcome the current limitations in mechanical performance of nanocelluloses. Structures composed of native elementary cellulose fibrils, made available by our approaches, is expected to further the developments and implementation of materials from sustainable building blocks. We expect that the introduced methods will push the upper strength 
boundaries measured for nanocelluloses, and their implementation in a wider range of applications.

\section{Acknowledgements}

We thank Dr. Markus Bacher for his support with NMR measurements, respectively. This research was funded in whole, or in part, by the Austrian Science Fund (FWF) (J4356). For the purpose of open access, the author has applied a CC BY public copyright license to any Author Accepted Manuscript version arising from this submission. The authors thank the financial support from the Austrian Biorefinery Centre Tulln (ABCT), the Academy of Finland (Project \# 311255, 'WTF-Click-Nano') and the H2020-ERC-2017-Advanced Grant 'BioELCell' (788489).

\section{References}

(1) Tardy, B. L.; Mattos, B. D.; Otoni, C. G.; Beaumont, M.; Majoinen, J.; Kämäräinen, T.; Rojas, O. J. Deconstruction and Reassembly of Nature's Supramolecular Structures into Next Generation Biobased Materials. Chem. Rev. 2021, in revision.

(2) Saito, T.; Kuramae, R.; Wohlert, J.; Berglund, L. A.; Isogai, A. An Ultrastrong Nanofibrillar Biomaterial: The Strength of Single Cellulose Nanofibrils Revealed via Sonication-Induced Fragmentation. Biomacromolecules 2013, 14 (1), 248-253. https://doi.org/10.1021/bm301674e.

(3) Li, T.; Chen, C.; Brozena, A. H.; Zhu, J. Y.; Xu, L.; Driemeier, C.; Dai, J.; Rojas, O. J.; Isogai, A.; Wlaagberg, L. Developing Fibrillated Cellulose as a Sustainable Technological Material. Nature 2021, 590 (7844), 47-56.

(4) Diddens, I.; Murphy, B.; Krisch, M.; Müller, M. Anisotropic Elastic Properties of Cellulose Measured Using Inelastic X-Ray Scattering. Macromolecules 2008, 41 (24), 9755-9759. https://doi.org/10.1021/ma801796u.

(5) Sakurada, I.; Nukushina, Y.; Ito, T. Experimental Determination of the Elastic Modulus of Crystalline Regions in Oriented Polymers. J. Polym. Sci. 1962, 57 (165), 651-660. https://doi.org/10.1002/pol.1962.1205716551.

(6) Šturcová, A.; Davies, G. R.; Eichhorn, S. J. Elastic Modulus and Stress-Transfer Properties of Tunicate Cellulose Whiskers. Biomacromolecules 2005, 6 (2), 10551061.

(7) Mattos, B. D.; Tardy, B. L.; Rojas, O. J. Accounting for Substrate Interactions in the Measurement of the Dimensions of Cellulose Nanofibrils. Biomacromolecules 2019, 20 (7), 2657-2665.

(8) Yang, X.; Reid, M. S.; Olsén, P.; Berglund, L. A. Eco-Friendly Cellulose Nanofibrils Designed by Nature: Effects from Preserving Native State. ACS Nano 2019, 14 (1), 724-735.

(9) Isogai, A.; Bergström, L. Preparation of Cellulose Nanofibers Using Green and Sustainable Chemistry. Curr. Opin. Green Sustain. Chem. 2018, 12, 15-21. https://doi.org/10.1016/j.cogsc.2018.04.008. 
(10) Plappert, S. F.; Nedelec, J.-M.; Rennhofer, H.; Lichtenegger, H. C.; Liebner, F. W. Strain Hardening and Pore Size Harmonization by Uniaxial Densification: A Facile Approach toward Superinsulating Aerogels from Nematic Nanofibrillated 2,3-

Dicarboxyl Cellulose. Chem. Mater. 2017, 29 (16), 6630-6641.

https://doi.org/10.1021/acs.chemmater.7b00787.

(11) Okita, Y.; Saito, T.; Isogai, A. Entire Surface Oxidation of Various Cellulose Microfibrils by TEMPO-Mediated Oxidation. Biomacromolecules 2010, 11 (6), 16961700. https://doi.org/10.1021/bm100214b.

(12) Kobayashi, Y.; Saito, T.; Isogai, A. Aerogels with 3D Ordered Nanofiber Skeletons of Liquid-Crystalline Nanocellulose Derivatives as Tough and Transparent Insulators. Angew. Chem. Int. Ed. 2014, 10394-10397. https://doi.org/10.1002/anie.201405123.

(13) Saito, T.; Kimura, S.; Nishiyama, Y.; Isogai, A. Cellulose Nanofibers Prepared by TEMPO-Mediated Oxidation of Native Cellulose. Biomacromolecules 2007, 8 (8), 2485-2491. https://doi.org/10.1021/bm0703970.

(14) Saito, T.; Hirota, M.; Tamura, N.; Kimura, S.; Fukuzumi, H.; Heux, L.; Isogai, A. Individualization of Nano-Sized Plant Cellulose Fibrils by Direct Surface Carboxylation Using TEMPO Catalyst under Neutral Conditions. Biomacromolecules 2009, 10 (7), 1992-1996. https://doi.org/10.1021/bm900414t.

(15) Saito, T.; Hirota, M.; Tamura, N.; Isogai, A. Oxidation of Bleached Wood Pulp by TEMPO/NaClO/NaClO2 System: Effect of the Oxidation Conditions on Carboxylate Content and Degree of Polymerization. J. Wood Sci. 2010, 56 (3), 227-232. https://doi.org/10.1007/s10086-009-1092-7.

(16) Fang, Z.; Li, B.; Liu, Y.; Zhu, J.; Li, G.; Hou, G.; Zhou, J.; Qiu, X. Critical Role of Degree of Polymerization of Cellulose in Super-Strong Nanocellulose Films. Matter 2020, 2 (4), 1000-1014. https://doi.org/10.1016/j.matt.2020.01.016.

(17) Pattinson, S. W.; Hart, A. J. Additive Manufacturing of Cellulosic Materials with Robust Mechanics and Antimicrobial Functionality. Adv. Mater. Technol. 2017, 2 (4), 1600084. https://doi.org/10.1002/admt.201600084.

(18) Beaumont, M.; Winklehner, S.; Veigel, S.; Mundigler, N.; Gindl-Altmutter, W.; Potthast, A.; Rosenau, T. Wet Esterification of Never-Dried Cellulose: A Simple Process to Surface-Acetylated Cellulose Nanofibers. Green Chem. 2020, 22, 56055609. https://doi.org/10.1039/D0GC02116D.

(19) Beaumont, M.; Jusner, P.; Gierlinger, N.; W. T. King, A.; Potthast, A.; Rojas, O. J.; Rosenau, T. Unique Reactivity of Nanoporous Cellulosic Materials Mediated by Surface-Confined Water. Nat. Commun. 2021, 12, 2513. https://doi.org/10.1038/s41467-021-22682-3.

(20) Beaumont, M.; Otoni, C. G.; Mattos, B. D.; Koso, T. V.; Abidnejad, R.; Zhao, B.; Kondor, A.; W. T. King, A.; Rojas, O. J. Regioselective and Water-Promoted Surface Esterification of Never-Dried Cellulose Fibers Towards Nanofibers with Adjustable Surface Energy. 2021. https://doi.org/10.26434/chemrxiv.14401712.v1.

(21) Mittal, N.; Ansari, F.; Gowda.V, K.; Brouzet, C.; Chen, P.; Larsson, P. T.; Roth, S. V.; Lundell, F.; Wågberg, L.; Kotov, N. A.; Söderberg, L. D. Multiscale Control of Nanocellulose Assembly: Transferring Remarkable Nanoscale Fibril Mechanics to Macroscale Fibers. ACS Nano 2018, 12 (7), 6378-6388. https://doi.org/10.1021/acsnano.8b01084.

(22) Beaumont, M.; Potthast, A.; Rosenau, T. Cellulose Nanofibrils: From Hydrogels to Aerogels. In Cellulose Science and Technology; Rosenau, T., Potthast, A., Hell, J., Eds.; John Wiley \& Sons, Inc.: Hoboken, NJ, USA, 2018; pp 277-339. https://doi.org/10.1002/9781119217619.ch13.

(23) Koso, T.; Rico del Cerro, D.; Heikkinen, S.; Nypelö, T.; Buffiere, J.; Perea-Buceta, J. E.; Potthast, A.; Rosenau, T.; Heikkinen, H.; Maaheimo, H.; Isogai, A.; Kilpeläinen, I.; 
King, A. W. T. 2D Assignment and Quantitative Analysis of Cellulose and Oxidized Celluloses Using Solution-State NMR Spectroscopy. Cellulose 2020, 27 (14), 79297953. https://doi.org/10.1007/s10570-020-03317-0.

(24) King, A. W. T.; Mäkelä, V.; Kedzior, S. A.; Laaksonen, T.; Partl, G. J.; Heikkinen, S.; Koskela, H.; Heikkinen, H. A.; Holding, A. J.; Cranston, E. D.; Kilpeläinen, I. LiquidState NMR Analysis of Nanocelluloses. Biomacromolecules 2018, 19 (7), 2708-2720. https://doi.org/10.1021/acs.biomac.8b00295.

(25) Wickholm, K.; Larsson, P. T.; Iversen, T. Assignment of Non-Crystalline Forms in Cellulose I by CP/MAS 13C NMR Spectroscopy. Carbohydr. Res. 1998, 312 (3), 123 129. https://doi.org/10.1016/S0008-6215(98)00236-5.

(26) Nocanda, X.; Larsson, P. T.; Spark, A.; Bush, T.; Olsson, A.; Madikane, M.; Bissessur, A.; Iversen, T. Cross Polarisation/Magic Angle Spinning 13C-NMR Spectroscopic Studies of Cellulose Structural Changes in Hardwood Dissolving Pulp Process. Holzforschung 2007, 61 (6). https://doi.org/10.1515/HF.2007.095.

Table of contents graphic

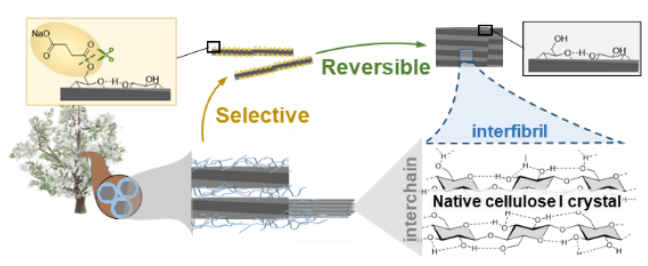

